retrospective series have reported skin adverse events under both allopurinol and febuxostat.

Objectives: To assess the cutaneous safety of febuxostat when used in patients with previous skin reactions to allopurinol.

Methods: A multicentre retrospective, descriptive study performed in seven Rheumatology units around Spain. Crystal-proven gout patients with previous CAR to allopurinol and treatment with febuxostat were selected. Demographic (age, gender), clinical (skin events, liver disease, concomitant thiazides) and laboratory variables (serum urate (SU), glomerular filtration rate) were collected. The primary study variable was the rate of patients developing CAR also with febuxostat. A descriptive analysis with estimation of $95 \%$ confidence interval $(95 \% \mathrm{Cl})$ is presented.

Results: Sixty-seven gout patients with prior allopurinol-related CAR treated with febuxostat were enrolled. Their average age was 68.1 years $(S D \pm 14.8)$, being 49 males $(73.1 \%)$. Thirteen of them were under thiazide treatment $(19.4 \%)$ and average glomerular filtration rate was $66.1 \mathrm{ml} / \mathrm{min}$ $( \pm 24.3)$ when allopurinol was started, at a median dose of allopurinol of $100 \mathrm{mg} /$ day (IQR 50-300) and mean SU of $8.9 \mathrm{mg} / \mathrm{dl}( \pm 1.7)$. The reported CAR under allopurinol were nonspecific in 55 (82.1\%), maculopapular rash in 9 (13.4\%), and Stevens-Johnson's syndrome in $3(4.5 \%)$. Out of 67 patients, 10 developed CAR with febuxostat $(14.9 \%$; $95 \% \mathrm{Cl}$ 8.3-25.3\%): nonspecific in 8 cases, one case of maculopapular rash and other of Stevens-Johnson's syndrome. Median (IQR) glomerular filtration rate, starting dose of febuxostat and SU level were 69.5 (42-87.8), 80 $\mathrm{mg} /$ day $(40-80)$ and $8.2 \mathrm{mg} / \mathrm{dl}(7.15-9.14)$, respectively.

Benzbromarone was initiated in all 10 patients with CAR to both allopurinol and febuxostat, and only one developed a nonspecific rash.

Conclusion: In this multicentre study, around $15 \%$ of patients with prior allopurinol-related CARs also developed them with febuxostat. Further prospective and intervention studies are needed to confirm these results, though caution is recommended when using febuxostat in this subgroup of patients.

Disclosure of Interests: Neus Quilis Marti: None declared, Paloma VelaCasasempere Grant/research support from: UCB, Abbvie, Pfizer, Roche, Bristol-Myer-Squibb (another research, not BIOBADASER related), Consultant for: UCB, Lilly, Pfizer, Roche, Bristol-Myer-Squibb, Speakers bureau: Roche, UCB, MSD, Pfizer, GSK, BMS, Lilly, Boris Anthony Blanco Cáceres: None declared, Cesar Diaz Torne Consultant for: Grünenthal, Speakers bureau: Grünenthal, Enrique Calvo-Aranda Speakers bureau: Grühnental, SOBI, Menarini, Francisca Sivera: None declared, Alejandro Prada-Ojeda: None declared, Fernando Perez-Ruiz Grant/research support from: Asociación reumatólogos de Cruces, Consultant for: Grünenthal Horizon Menarini, Speakers bureau: Grünenthal, Menarini, Fundación Española Reumatologiá, Eliseo Pascual Consultant for: Eliseo Pascual has participated in the boards of Astra Zeneca, Menarini, Grunenthal and Horizon, Speakers bureau: speaking fees from Astra Zeneca and Grunenthal, Mariano Andres: None declared

DOI: 10.1136/annrheumdis-2019-eular.4394

\section{SAT0442 CALCIUM PYROPHOSPHATE CRYSTAL ARTHRITIS DURING HOSPITALIZATIONS: A PROSPECTIVE, CRYSTAL-PROVEN CASE SERIES}

Laura Ranieri ${ }^{1}$, Francisca Sivera ${ }^{2}$, Mariano Andrés ${ }^{1} .{ }^{1}$ Alicante, Hospital General Universitario Alicante, Alicante, Spain, ${ }^{2}$ Hospital General Universitario de Elda, ALICANTE, Spain

Background: Despite having passed more than fifty years after its initial description, essential questions for calcium pyrophosphate (CPP) crystal disease, such as clinical spectrum, diagnosis or management schemes, remain unsolved. Acute flares often occurred during hospitalizations. Scant reports have addressed this common setting for CPP crystal disease, and whether these patients behave similarly to ambulatory cases is unknown.

Objectives: The aim of this work was to describe in a prospective way a crystal-proven case series of patients developing acute CPP crystal arthritis during hospitalizations for another conditions.

Methods: An observational, cross sectional descriptive study was conducted in two Spanish centers from November 2013 to December 2018. A prospective convenience sampling was employed to select patients with crystal-proven CPP acute arthritis seen during hospital admissions. Demographic, clinical and CPP-related variables were collected, and X-rays (pelvis, knees, hands, affected joint when different) and laboratory tests (to rule out associated metabolic conditions) were systematically requested. A descriptive analysis is presented.

Results: 90 episodes of acute CPP arthritis in 87 patients were seen in the study period, with an average age of 81.8 years (SD 7.7), 50.6\% of them men. Approximately $26 \%$ of patients reported prior episodes of arthritis, most of them $(68.4 \%)$ as outpatients. Only three patients were on preventive treatment for CPP arthritis (two on colchicine and one on low dose glucocorticoids). Regarding the acute CPP arthritis during admissions, they were predominantly monoarticular $(81.0 \%)$ and the main involved joints were knee $(46.0 \%)$, wrist $(13.8 \%)$ and ankles $(6.9 \%)$. The reasons for admission were diverse, with a mean of 7.7 days (SD 9.1) from admission to flare. About X-rays, $23.8 \%$ showed no chondrocalcinosis (CC) in the evaluated joints [61/80]. In $57.1 \%$ of patients there was chondrocalcinosis in the affected joint [44/77] and regarding usual joints: $74.3 \%$ in knees [55/74], $51.5 \%$ in triangular carpal ligament [34/66], $25.4 \%$ in metacarpo-phalangeal joints [17/67], $20 \%$ in pubic symphysis [14/70] and $17.6 \%$ in coxofemoral joints [12/68]. A secondary form of osteoarthritis was only seen in 10 patients (12.5\%). About associated metabolic diseases, one case of primary hyperparathyroidism-related hypercalcemia and five cases of hypomagnesemia at the time of the flare were detected. In all six patients with a polyarticular presentation, laboratory tests for rheumatoid factor and ACPA were negative.

Conclusion: From the findings of this prospective, crystal-proven series of CPP crystal arthritis in an intrahospital setting, we can remark:

- 1. The low numbers of previous ambulatory flares may suggest a different clinical entity of CPP disease.

- 2. Radiological CC was absent in around a quarter of patients despite a extensive assessment, so synovial fluid analysis remains essential for diagnosis.

- 3. The rarity of associated metabolic diseases seen runs against ruling out secondary causes of CPP disease in this setting.

Disclosure of Interests: None declared

DOI: 10.1136/annrheumdis-2019-eular.7407

\section{SAT0443 RECONCILIATION OF URATE LOWERING THERAPIES DURING HOSPITALIZATION AND THE IMPACT OF RHEUMATOLOGIC CONSULTATION ON MANAGEMENT OF INPATIENT GOUT FLARES}

Mitali Sen. Emory University, Rheumatology, Atlanta, United States of America

Background: Hospitalizations complicated by gout flares have an impact on patient care. Delayed diagnosis and suboptimal management can lead to prolonged discomfort, impair chronic outcomes of the disease and lengthen the hospitalization (1). Patients on urate lowering therapy (ULT) are frequently admitted to the hospital for unrelated causes but there is variability in inpatient medication management of the urate lowering agent and acute management of the flare.

Objectives: In this descriptive study, we analyse the variability of reconciliation of ULT on admission and discharge and the impact of rheumatology consultation on acute and chronic management of gout.

Methods: Patients- above the age of 18- admitted to our tertiary care hospital from $01 / 01 / 2010$ to $01 / 01 / 2016$ with an ICD-9 or an ICD-10 diagnosis of gout were reviewed. The first 200 patients underwent a retrospective chart review as a pilot study for an ongoing project. We reviewed patient demographics, laboratory testing, and co-morbid conditions; medications on admission and discharge, incidence of gout flarediagnosis and management during hospitalization, rheumatology consultation and discharge plan for these patients.

Results: Of the 200 patients reviewed, 2.69 admissions per person. We further described the patients who had a gout flare during hospitalization ( $n=54,27 \%$ ). $66 \%$ of these patients were males, mean age 69.8 years and BMI $31.78 \mathrm{~kg} / \mathrm{m}^{2}$. A majority of patients had hypertension, renal disease, and dyslipidemia (Table 1).

$70 \%$ of the patients were on chronic medications for gout (Table 2) $29.6 \%$ of these patients were continued on these agents upon admission and only $64.8 \%$ of these patient was eventually discharged on these drugs.

Rheumatology consulted for $68.5 \%$ of the patients. Arthrocentesis was more frequently performed when rheumatology was consulted $(70 \%$ vs.17.6\%; $p<0.001)$. Rheumatology consultation did not decrease length of stay in the hospital. $78.5 \%$ of the patients managed by primary team were discharged on a ULT or colchicine compared to $100 \%$ in the group managed by rheumatology consult team $(100 \%$ vs $78.5 \%$; $<<0.0431)$. Outpatient rheumatology follow up was documented in discharge papers for $62 \%$ of the patients managed with rheumatology consult compared to $11.7 \%$ in the comparison group. (62\% vs $11.7 \%$; $<0.002$ ).

Conclusion: Rheumatology consultation improved adherence to guidelines in diagnosis and management of gout flare and improved the discharge planning and follow up. 